\section{Arrea: Ginecalogia \\ REVIEWING THE GUIDELINES FROM THE Brazilian Ministry of Health fOR DIAGNOSIS OF EARLY PREGNANCY}

\section{INTRODUCTION}

Identification of early pregnancy is very important. Some medications are not safe during pregnancy and should be avoided. In addition, complications related to ruptured ectopic pregnancy or miscarriage are major causes of mortality among Latin American women $^{1}$. A urine pregnancy test for all patients in reproductive age experiencing pelvic pain or vaginal bleeding has been recommended $^{2,3}$. Nevertheless, the Brazilian Health Ministry recommends that a pregnancy test should be ordered when the patient refers amenorrhea or a menstrual delay ${ }^{4}$. Likewise, the guidelines from the Brazilian Medical Association for the prenatal care state a grade $D$ recommendation for the use of urinary $b-h_{C G}{ }^{5}$ test. Although the accuracy of gynecologic exam for ectopic pregnancy, is well established in the literature, in Brazil, little is known about the accuracy of gynecologic physical examination to diagnose any type of pregnancy. Similarly, there is no data about the prevalence of pregnant women in the ER (Emergency Room) without the diagnosis of gestation. After searching the medical literature ((("Pregnancy"[Mesh] OR "Pregnancy, Unplanned"[Mesh])) AND ("Emergency Service, Hospital"[Mesh] OR "Emergency Medical Services"[Mesh])) AND "Prevalence"[Mesh] from 1966 to June 2010) 9 articles were found. Of these, only one article was found about the prevalence of unrecognized pregnancy in the emergency department ${ }^{6}$. The authors demonstrated that clinical history can be used to determine which patient requires a pregnancy test, when one of the following risk factors is present: abdominal/pelvic complaints, abnormal last menstrual period or patient suspects being pregnant. ${ }^{6}$ However, the Brazilian Ministry of Health and the Brazilian Medical Association guidelines do not follow this recommendation and the accuracy of gynecologic physical exam for diagnosis of early pregnancy, compared to the urine pregnancy test in patients with one of these risk factors is not known. This study intends to provide evidence of the accuracy of gynecological examination for diagnosis of early pregnancy during consultation in the emergency room where patients and gynecologists are not aware of the pregnant condition.. Moreover, the prevalence of unrecognized pregnancy among women in reproductive age was identified in the ED. The primary outcomepurpose was to analyze the pre-test probability, sensitivity, specificity, positive and negative predictive values and likelihood ratios of clinical history and physical examination for pregnancy diagnosis when compared to b-hCG. The secondary purposeoutcome was to stratify the accuracy among experienced (professor and medical staff) and in-training personnel (residents, fourth and fifth year medical students). With these data, the Brazilian health authorities can establish safer protocols based on evidence.

\section{Methods}

This study was carried out at the ER of Hospital de Clínicas de Porto Alegre (a tertiary teaching hospital), from August 2007 to August 2008. Women in reproductive age, (between 14 and 50 years old) with abdominal pain and/or abnormal uterine bleeding were eligible for the study. Menopausal women, women with a prior hysterectomy and those with a known pregnancy were excluded. Recruitment of the study population was based on selection criteria, during one entire year to reflect the full spectrum of patients.

After medical consultation, those who met study criteria and provided consent were invited to participate. After clinical examination, the attending health provider (professor, staff physician, resident, or medical student) recorded his/her opinion on pregnancy status in a spreadsheet. All patients were submitted to a bimanual exam and speculum examination was performed at the health provider's discretion. Following consultation, a urinary pregnancy test (hCG Strip Test Plus, ALAMAR, SP, Brazil) was ordered and sent to the central laboratory. Clinical and laboratory tests were performed on all patients. The test was performed within 3 hours by certified technicians who were not aware of the clinical opinion of the health provider.

The sample size was calculated according to literature ${ }^{7}$ including the variables: an expected sensitivity and specificity of $95 \%$, the prevalence of pregnant women without the diagnosis of pregnancy in the ER as $20 \%$ and a lower 95 than $95 \%$ confidence interval of $>75 \%$, in an expected proportion for a dichotomousic variable. This calculation showed that at least 34 cases and 136 controls should be found, yielding a total of 170 patients.

Statistical analysis was done in a $2 \times 2$ contingency table in order to obtain the sensitivity, specificity, post-test probabilities (positive and negative predictive values), and positive and negative likelihood ratios with their 95\% confidence intervals. Calculations were performed using GraphPad InStat version 3.0a for Macintosh (GraphPad Software, San Diego California, USA) and the web based Diagnostic Test Calculator (http://araw.mede. uic.edu/cgi-bin/testcalc. pl?DT $=0 \& D t=0 \& d T=0 \& d t=0 \& 2 \times 2=C$ ompute)

This study was approved by the ethics committee of Hospital de Clínicas de Porto Alegre.

\section{RESULTS}

From August 2007 until August 2008, 4124 patients came to the gynecologic emergency room. Of these, 3723 were in reproductive age and 320 of them had pain or vaginal bleeding as their main complaint. One hundred and twenty of these patients did not meet inclusion criteria. We analyzed the results of the remaining 200 patients who satisfied the inclusion criteria and completed the clinical evaluation and laboratory pregnancy test. The prevalence of pregnancy in our sample population was $18.5 \%$ (37/200). The median gestational age at time of diagnosis was 7 weeks (range 5- 20 weeks). Further details of the study are depicted in Figure 1 (flow chart). Test results were obtained within 3 hours. Because of the nature of the index test (gynecological 
examination) no reproducibility studies were not donemade. None of the patients had a gestational age $\geq 20$ weeks. Seven pregnancies were missed by the clinical exam. Two were cases of ectopic pregnancies at 6 and 7 weeks and the other 5 false positive diagnoses were in patients who had leiomyomata uteri. Over a 12 month period, 182 curettages for miscarriage and 16 insurgeries casesof ectopic pregnancies were performed. Results are summarized in Table 1.

\section{Discussion}

A frequent belief among practitioners in clinical medicine is that laboratory tests and imaging techniques are more definitive than medical history and examinations ${ }^{8}$. Some authors advocate that it is unnecessary to perform physical examination in a suspected case of ectopic pregnancy ${ }^{9}$. NeverthelessHowever, some cases of ectopic pregnancy can be missed, even with a negative urine ${ }^{10}$ or blood test ${ }^{11}$. LikewiseAs, a presumed diagnosis of ectopic pregnancy is inaccurate in almost $40 \%$ of cases $^{12}$.
In the clinical setting of an emergency room, the main concern is to miss the diagnosis of ectopic pregnancy and send the patient home. This, of course may have greater significance than to miss diagnosis of a threatened abortion. The former requires treatment while the latter requires observation. Another problem could be prescription of a class $\mathrm{C}$ antibiotic in the first trimester of pregnancy.

This study provides evidence about the accuracy of clinical gynecologic examination performed by specialists, residents and medical students to diagnose early pregnancy and the prevalence of unrecognized pregnancy. The strength of this study is its spectrum of patients, reflecting as close as possible the emergency room reality. The majority of patients waswere in the first trimester of pregnancy. The eExperience of the examiners varied. Clinical assessments were performed carried out by one professor, 7 staff physicians, 14 residents and 10 medical students on a rotation schedule. No standard questionnaire was used for the clinical examination,examination; however the sensitivity found in our study, $81 \%$, was similar to thate one reported by Stengel et al. 6 , who found $83 \%$. The dDate of the last menstrual period and the use or not of contraceptive methods were questions included

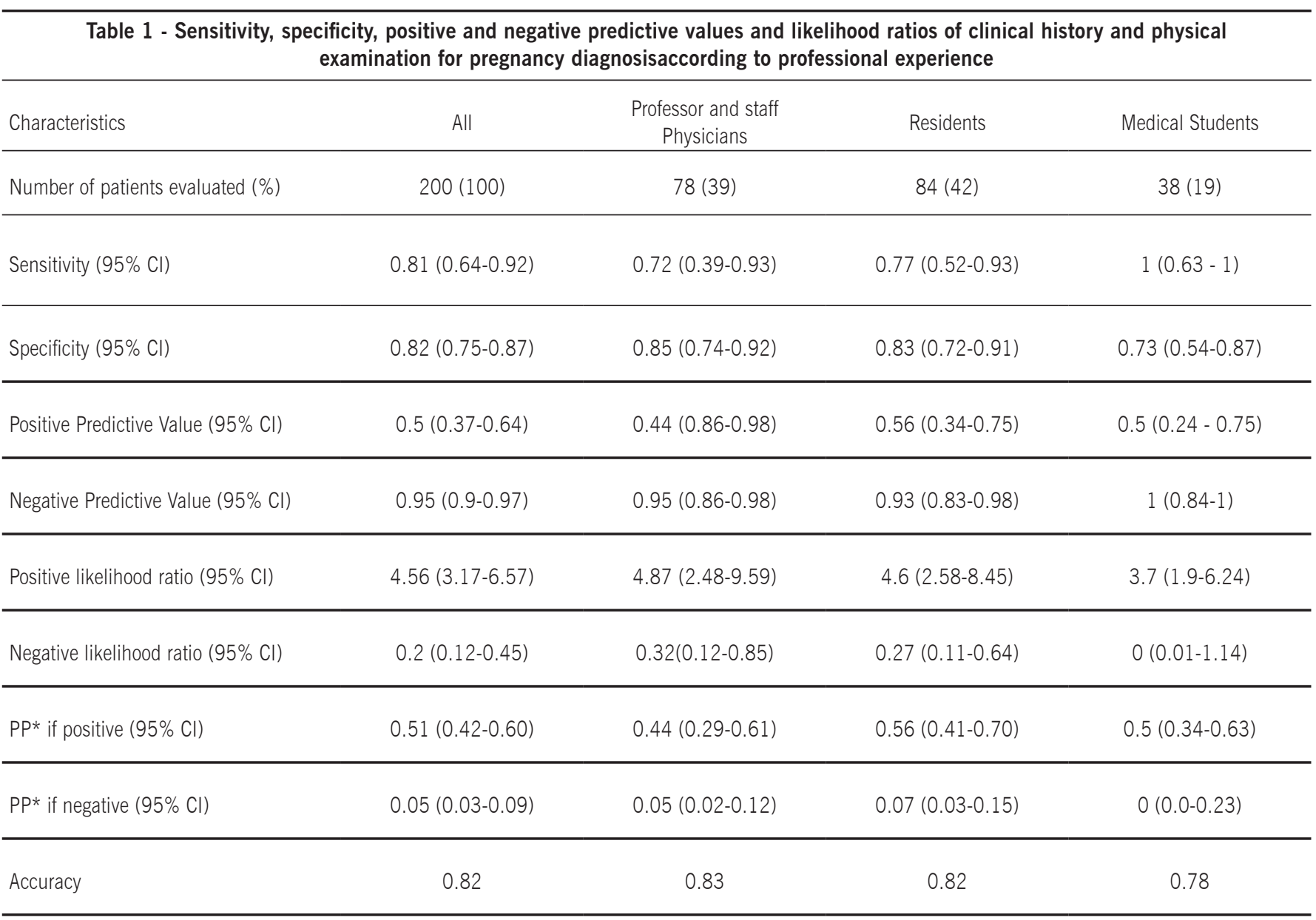

* PP: Posterior probability 
in the history. These questions are considered reliable for ruling out pregnancy ${ }^{13}$, although other authors did not find this correlation $^{6}$. Bimanual pelvic examination was performed in all cases. Doppler sonar, for detection of fetal cardiac activity, and speculum examination were performed at the health provider's discretion. According to Hoey and Allan, the routine use of speculum exam plays a role in a minority of management decisions ${ }^{14}$.

The serum b-hCG has been considered the gold standard for pregnancy diagnosis, however, the reference standard test used in this study has a sensitivity of $25 \mathrm{mlU} / \mathrm{mL}$ of $\mathrm{b}-\mathrm{hCG}$ in urine and this exam has a high accuracy, compared to the blood b-hCG. A negative result after one week after the expected date of the missed period virtually guarantees that the woman is not pregnant $15,16,16$. This test is approved by ANVISA, the Brazilian agency equivalent to the American Food and Drug Administration. The reference standard test was applied to all patients in a blinded fashionway. The rResults depicted in Table 1 give us evidence of the performance of the clinical examination for diagnosis of early pregnancy, and the prevalence of pregnancy in women, without the diagnosis of pregnancy, who were attended at the emergency room due for to pelvic pain or irregular bleeding (18.5\%). The oOverall accuracy of the clinical examination was around $80 \%$.

Of greater interest was the finding that clinical examination is not very accurate for identifying pregnancy. The positive likelihood ratio was under 5 , given an overall post-test probability of $51 \%$, which is similar to the results found by Stovall et al. for diagnosis of ectopic pregnancy. ${ }^{17}$ When the overall examiners assumed that the patient was not pregnant, they were wrong in only 5\% (95\% $\mathrm{Cl}$ 0.03-0.09). At first, this seems to be an excellent performance for a clinical exam. However, in 7 false negative cases, i.e., clinical exam was negative, and the urine pregnancy test was positive, 2 cases of ectopic pregnancy were missed. For clinical policies, it is important to know the prevalence of certain conditions, before using the test; this is called the pretest probability. Based on that, the likelihood ratio can be calculated. To the best of our knowledge, this information about the Brazilian reality is not available in literature and it reflects a common clinical scenario, which may be used as a reference for other Brazilian health authorities.

\section{Conclusion}

In conclusion, after clinical gynecological examination we were able to exclude or confirm early pregnancy in 91-97\%, and $42-60 \%$ of the cases, respectively, yielding an accuracy of $82 \%$. However, due to potential risks of an ectopic pregnancy, a urine pregnancy test should be used to confirm or exclude early pregnancy, regardless of the absence, delay of menses or amenorrhea.

Ricardo Francalacci Savaris ${ }^{1 *}$ Gisele Silva de Moraes ${ }^{2}$

Rafael do Amaral Cristovam ${ }^{3}$

1. Doutorado em Ciências Médicas - Professor adjunto da Universidade Federal do Rio Grande do Sul e professor do serviço de ginecologia e obstetrícia do Hospital de Clínicas de Porto Alegre, Porto Alegre, RS
2. Acadêmica do sexto ano de Medicina; bolsista de iniciação em pesquisa CNPq, Porto Alegre, RS

3. Acadêmico do sexto ano de Medicina da Universidade Federal do Rio Grande do Sul, Porto Alegre, RS

Study conducted at Universidade Federal do Rio Grande do Sul, Departamento de Ginecologia e Obstetrícia, Porto Alegre, RS

\section{*Correspondence:}

Universidade Federal do Rio Grande do Sul

Departamento de Ginecologia e Obstetrícia

Rua Ramiro Barcelos, 2350/1125 - Porto Alegre, RS

CEP: 90035-903

\section{Financial Support: FIPE (06-300)}

\section{References}

1. Khan K S, Wojdyla D, Say L, Gulmezoglu A M, Van Look P F. WHO analysis of causes of maternal death: a systematic review. Lancet. 2006;367:1066-74. 2. Minaretzis D, Tsionou C, Tsandoulas E. Clinical evaluation of a sensitive urine pregnancy test in the gynecological emergencies. Eur J Obstet Gynecol Reprod Biol. 1991;39:123-6.

3. Close R. Abdominal and pelvic pain in the nonpregnant patient. In: Tintinalli JE, Kelen GD, Stapczynski JS, editors. Emergency medicine. Philadelphia: McGraw-Hill; 2003.

4. Brasil. Ministério da Saúde. Pré-natal e puerpério: atenção qualificada e humanizada - manual técnico / Ministério da Saúde, Secretaria de Atenção à Saúde, Departamento de Ações Programáticas Estratégicas. Brasília (DF): Ministério da Saúde; 2005.

5. Associação Médica Brasileira, Conselho Federal de Medicina. Projeto Diretrizes. Assistência Pré-Natal. [citado jun 2006]. Disponível em: http://www. projetodiretrizes.org.br/5_volume/02-AssistPre.pdf

6. Stengel CL, Seaberg DC, MacLeod BA. Pregnancy in the emergency department: risk factors and prevalence among all women. Ann Emerg Med. 1994;24:697-700. 7. Flahault A, Cadilhac M, Thomas G. Sample size calculation should be performed for design accuracy in diagnostic test studies. J Clin Epidemiol. 2005:58:859-62.

8. Halkin A, Reichman J, Schwaber M, Paltiel O, Brezis M. Likelihood ratios: getting diagnostic testing into perspective. QJM. 1998;91:247-58.

9. Mol BW, Hajenius PJ, Engelsbel S, Ankum WM, Van der Veen F, Hemrika DJ, et al. Should patients who are suspected of having an ectopic pregnancy undergo physical examination? Fertil Steril. 1999;71:155-7.

10. Kalinski MA, Guss DA. Hemorrhagic shock from a ruptured ectopic pregnancy in a patient with a negative urine pregnancy test result. Ann Emerg Med. 2002;40:102-5.

11. Maccato M L, Estrada R, Faro S. Ectopic pregnancy with undetectable serum and urine beta-hCG levels and detection of beta-hCG in the ectopic trophoblast by immunocytochemical evaluation. Obstet Gynecol. 1993;81:878-80. 12. Barnhart K T, Katz I, Hummel A, Gracia C R. Presumed diagnosis of ectopic pregnancy. Obstet Gynecol. 2002;100:505-10.

13. Bastian LA, Piscitelli JT. Is this patient pregnant? Can you reliably rule in or rule out early pregnancy by clinical examination? JAMA. 1997;278:586-91. 14. Hoey R, Allan K. Does speculum examination have a role in assessing bleeding in early pregnancy? Emerg Med J. 2004;21:461-3.

15. Chard T. Pregnancy tests: a review. Hum Reprod. 1992;7:701-10.

16. Olshaker JS. Emergency department pregnancy testing. J Emerg Med. 1996;14:59-65.

17. Stovall TG, Kellerman AL, Ling FW, Buster JE. Emergency department diagnosis of ectopic pregnancy. Ann Emerg Med. 1990;19:1098-103. 